\title{
Responses of Human Anterior Cingulate Cortex Microdomains to Error Detection, Conflict Monitoring, Stimulus-Response Mapping, Familiarity, and Orienting
}

\author{
Chunmao Wang, ${ }^{1,2}$ Istvan Ulbert, ${ }^{2,5}$ Donald L. Schomer, ${ }^{3}$ Ksenija Marinkovic, ${ }^{2}$ and Eric Halgren ${ }^{2,4}$ \\ ${ }^{1}$ Comprehensive Epilepsy Center, New York University School of Medicine, New York, New York 10016, ${ }^{2}$ Massachusetts General Hospital/Massachusetts \\ Institute of Technology/Harvard Medical School Martinos Center for Biomedical Imaging, Charlestown, Massachusetts 02129, ${ }^{3}$ Department of Neurology, \\ Beth Israel Deaconess Medical Center, Harvard Medical School, Boston, Massachusetts 02215, ${ }^{4}$ Institut National de la Santé et de la Recherche Médicale, \\ Marseille 13009, France, and 5nstitute for Psychology, Hungarian Academy of Sciences, Budapest 1068, Hungary
}

\begin{abstract}
Human anterior cingulate cortex (ACC) activity modulation has been observed in numerous tasks, consistent with a wide variety of functions. However, previous recordings have not had sufficient spatial resolution to determine whether microdomains (approximately one to two columns) are involved in multiple tasks, how activity is distributed across cortical layers, or indeed whether modulation reflected neuronal excitation, inhibition, or both. In this study, linear arrays of 24 microelectrodes were used to estimate population synaptic currents and neuronal firing in different layers of ACC during simple/choice reaction time, delayed word recognition, rhyming, auditory oddball, and cued conditional letter-discrimination tasks. Responses to all tasks, with differential responses to errors, familiarity, difficulty, and orienting, were recorded in single microdomains. The strongest responses occurred $\sim 300-800$ ms after stimulus onset and were usually a current source with inhibited firing, strongly suggesting active inhibition in superficial layers during the behavioral response period. This was usually followed by a sink from $\sim 800$ to $1400 \mathrm{~ms}$, consistent with postresponse rebound activation. Transient phase locking of task-related theta activity in superficial cingulate layers suggested extended interactions with medial and lateral frontal and temporal sites. These data suggest that each anterior cingulate microdomain participates in a multilobar cortical network after behavioral responses in a variety of tasks.
\end{abstract}

Key words: intracranial EEG; current source density; unit firing; attention; memory, theta

\section{Introduction}

The anterior cingulate cortex (ACC) lies at the crossroads of three vast anatomo-functional systems: motor, limbic, and prefrontal (Vogt et al., 2004). In the motor system, the ACC lies immediately inferior to the supplementary motor cortex and projects to motor cortex as well as the spinal cord (Dum and Strick, 1993). In the limbic system, the ACC is directly related to the subicular complex (and thus hippocampus), posterior orbital cortex (area 32), and the anterior thalamic nucleus, as well as brainstem nuclei concerned with autonomic control. Finally, the ACC has strong bidirectional connections with the dorsolateral prefrontal and temporal cortices (Barbas, 2000; Vogt et al., 2004).

Consistent with these connections, functional magnetic resonance imaging (fMRI) and positron emission tomography (PET) have found ACC hemodynamic activation in a wide variety of tasks involving reading (Fiez and Petersen, 1998), word generation

Received Oct. 6, 2004; revised Nov. 19, 2004; accepted Nov. 26, 2004.

This work was supported by National Institutes of Health Grants NS18741 and NS44623. We thank G. Karmos, G. Heit, B. Rosen, J. R. Ives, and M. Glessner for their contributions to this research. This paper is dedicated to the memory of Howard Blume.

Correspondence should be addressed to Eric Halgren, Martinos Center for Biomedical Imaging, Six 13th Street, Charlestown, MA 02129. E-mail: halgren@nmr.mgh.harvard.edu.

DOI:10.1523/JNEUROSCI.4151-04.2005

Copyright $\odot 2005$ Society for Neuroscience $\quad$ 0270-6474/05/250604-10\$15.00/0
(Crosson et al., 1999), episodic recall (Nyberg, 1998; Cabeza et al., 2003), working memory (Bunge et al., 2001), emotion (Phan et al., 2002), and attention (Mesulam, 1981; Corbetta et al., 1998; Cabeza et al., 2003). ACC activation is related to the number of possible responses in a task, suggesting that it may contribute to response choice or "selection-for-action" (Posner et al., 1988; Petersen et al., 1989; Frith et al., 1991). This may reflect a basic contribution to motor control (Kollias et al., 2001; Picard and Strick, 2001) or a role in detecting situations that requires strategic intervention because of conflicting potential responses that may lead to errors (Carter et al., 2000; Kiehl et al., 2000).

Some authors emphasize the juxtaposition of different functions, especially emotional and cognitive as the key to ACC function (Duncan and Owen, 2000; Allman et al., 2001; Paus, 2001), whereas others emphasize the anatomical segregation of different functions (Peterson et al., 1999). For example, cognitive activation in ACC has been located anterior to the anterior commissure line of Talairach and posterior to the anterior limit of the corpus callosum, with emotional activation in more anteroinferior ACC (Bush et al., 2000). Motor areas may be above and behind the cognitive division (Dum and Strick, 2002), visuospatial areas in posterior cingulate, and memory-related areas inferior to posterior cingulate in retrosplenial cortex (Vogt et al., 2004).

As an alternative to anatomical segregation, some functions 


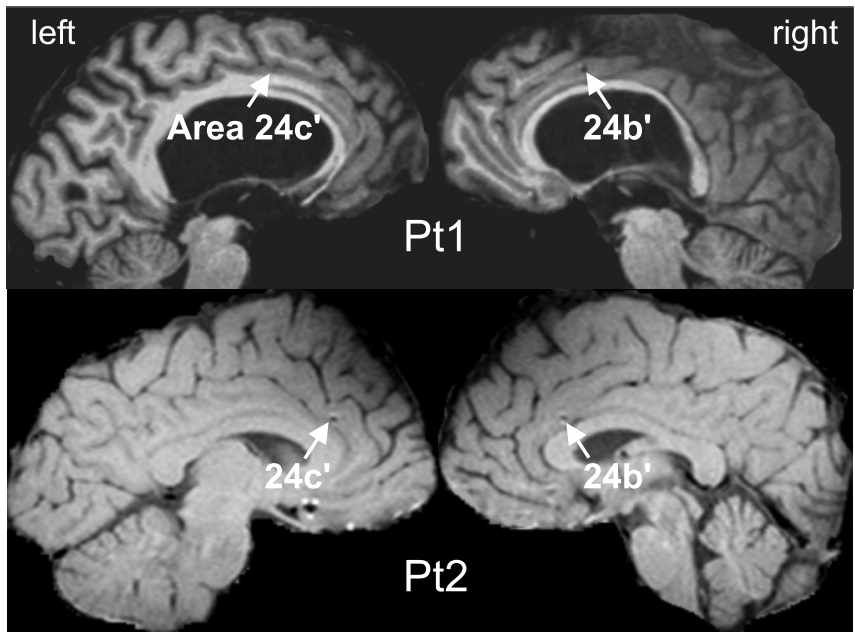

Figure 1. Locations of laminar probes in MRIs taken with the electrodes in situ. Laminar probes are indicated by dark MRI artifacts (arrowheads; artifacts are larger than the actual electrodes). All contacts appear to lie in Brodmann's area 24' (Vogt et al., 2004). Note the dilation of the third ventricle in Pt1 attributable to compensated aqueductal stenosis.

may reflect different levels of neuronal processing related to the same integrative function. For example, the ACC may contribute to both orienting-monitoring and response-choice but at different phases of the response or trial. This would imply that there is also a temporal segregation of ACC function.

It is difficult to distinguish between these hypotheses using noninvasive imaging methods because of their limited spatial resolution (Dale and Halgren, 2001). Furthermore, noninvasive methods lack the physiological resolution to distinguish excitation from inhibition (EEG and magnetoencephalography) or are hemodynamic, which has an unknown relationship to neuronal activity. Intracranial measures have greater spatial accuracy but typically record from all cortical layers in a region that contains many cortical columns. The current study used simultaneous recordings from an array of microelectrodes to characterize synaptic activity and neuronal firing in relatively small populations of ACC neurons. These data demonstrate that multiple responses related to novelty, memory, set, errors, and the difficulty of stimulus-response mapping are colocalized at a submillimeter level. Surprisingly, inhibition in superficial cortical layers appeared to be a common response by the ACC to cognitive stimuli. Relative excitation after the inhibition may provide a window when the ACC contributes to wider cortical processing.

\section{Materials and Methods}

Patients and electrodes. Patients with complex partial seizures resistant to all appropriate medications were considered for surgical removal of their epileptogenic focus. When extensive noninvasive diagnostic tests were unable to unambiguously locate the focus, intracranial recordings from potential seizure onset sites were recommended. Patient $1(\mathrm{Pt} 1)$ was a 35-year-old right-handed male; patient 2 (Pt2) was a 55-year-old righthanded female. Their intelligence and personality were in the normal range. Both gave fully informed consent according to National Institutes of Health guidelines to add a linear array of microcontacts to the tips of their clinical macroelectrodes. Each laminar probe was $\sim 3.5 \mathrm{~mm}$ long with a row of $2440-\mu \mathrm{m}$-diameter contacts separated by $110 \mu \mathrm{m}$ (Ulbert et al., 2001a). The choice of patients and sites to implant, as well as the duration of implantation, were made on completely clinical grounds. Successful recordings were obtained bilaterally from area $24^{\prime}$ of the ACC in both patients (Fig. 1). Electrode positions were slightly more posterior in patient 1 , rendering it possible that one or both could lie in the anterior cingulate motor area. However, there were no systematic response differences noted between patients. Seizure origin in Pt1 was multifocal in the right hemisphere including the hippocampus and oribitofrontal cortex and in Pt2 was in the left temporal lobe remote from the ACC; the ACC recordings reported here did not display interictal spikes during waking and were made at least $24 \mathrm{~h}$ after the most recent seizure.

Recordings. Differential recordings were made from 23 pairs of successive contacts. After wideband (DC, 10,000 Hz) preamplification (gain, $10 \times$; common mode rejection ratio, $90 \mathrm{db}$; input impedance, $10^{12} \Omega$ ), the signal was split into field potentials (filtered at $0.2-500 \mathrm{~Hz}$; gain, $1000 \times$; digitized at $2000 \mathrm{~Hz} ; 16 \mathrm{bit}$ ) and action potentials (filtered at $200-5000 \mathrm{~Hz}$; gain, $1000 \times$; digitized at $20,000 \mathrm{~Hz} ; 12 \mathrm{bit}$ ) and stored continuously with stimulus markers. Population transmembrane current flows were estimated using linear current source density (CSD) analysis (Nicholson and Freeman, 1975; Ulbert et al., 2001b), calculated using the second spatial derivative of local field potentials (LFP). Population action potentials [multiunit activity (MUA)] were estimated by rectifying the bandpass filtered data [zero phase shift, $300-3000 \mathrm{~Hz} ; 48$ $\mathrm{db} /$ octave (oct)] and then low-pass filtering the result (zero phase shift, $30 \mathrm{~Hz} ; 12 \mathrm{db} / \mathrm{oct})$.

Spatial resolution. A simulation was performed to evaluate how steeply LFP and CSD decline with distance from the neural generator (see supplemental material I, available at www.jneurosci.org). CSD amplitude in this simulation declines 10 -fold at $\sim 300 \mu \mathrm{m}$ from the cortical column, whereas potential decreases at a slower slope. This falloff is in the direction parallel to the cortical surface; the falloff is more rapid in the orthogonal direction, actually reaching zero while still within the cortex. Theoretical and empirical studies in animals indicate that MUA should decline with distance at least as rapidly as a result of the short duration, asynchrony, and spatial distribution of action potential fields (Humphrey, 1968; Grover and Buchwald, 1970). In summary, CSD and MUA estimate the activity of neurons in a volume approximately corresponding to that of a cortical column.

Spectral analysis. For time-frequency spectral measures, the single trial signal for each channel was convolved with complex Morlet's wavelets (Kronland-Martinet et al., 1987; Halgren et al., 2002). Relatively constant temporal and frequency resolution across target frequencies was obtained by adjusting the wavelet widths according to the target frequency. The wavelet widths increase linearly from 1 to 6.5 as frequency increases from 1 to $13 \mathrm{~Hz}$, resulting in a constant temporal resolution of $80 \mathrm{~ms}$ and frequency resolution of $2 \mathrm{~Hz}$. Tests with simulated data confirmed that the methods used here accurately measure spectral power patterns, even at frequencies as low as $1 \mathrm{~Hz}$ (see supplemental material II, available at www.jneurosci.org).

In addition to the microarray recordings in ACC, recordings from clinical macrocontacts were available in multiple temporal and frontal sites in Pt2. The macrocontacts consisted of 1.3-mm-diameter cylinders, each $1.5 \mathrm{~mm}$ long and separated from the next contact by $3.5 \mathrm{~mm}$. Our initial results showed that event-related spectral activity is mainly in the theta range and is generated in the superficial ACC layers. Thus, we focused our calculations on the theta and adjacent bands and calculated the phase locking between the superficial ACC contacts and simultaneous macrorecordings. This measure is sensitive to the similarity of the timing of activity in a particular frequency range between two structures, regardless of their amplitudes (Lachaux et al., 1999). Our simulation confirmed that the method accurately detects the phase-locking period, even at frequencies as low as $1 \mathrm{~Hz}$ (see supplemental material II, available at www.jneurosci.org). Potential gradients from both microrecordings and macrorecordings were used for these calculations. Statistical significance of the difference between conditions for a particular recording channel, latency, and measure (CSD, MUA, or spectral power) was assessed using a $t$ test of values from individual trials. Significant deviations of responses from baseline were assessed using one-sample $t$ tests of values from each trial. Threshold was set at $p<0.01$ (two-tailed). Time-frequency maps of spectral power or phase-locking factor are displayed as $z$-scores relative to the mean and variance of the same measure in the prestimulus period. Baseline measures were calculated separately for each frequency and channel.

Cognitive tasks. To probe ACC activity during different functions, we used the following five tasks (Fig. 2): (1) simple/choice reaction time 
Simple/Choice Reaction Time (Simple/Choice RT)

Simple- always press left

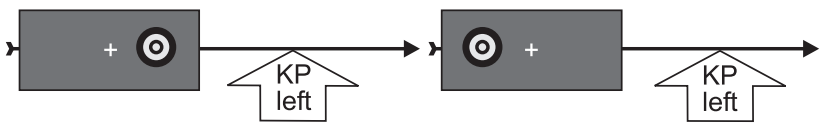

Choice- press ipsilateral to the stimulus

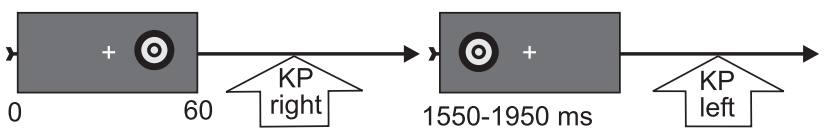

Simple- always press right

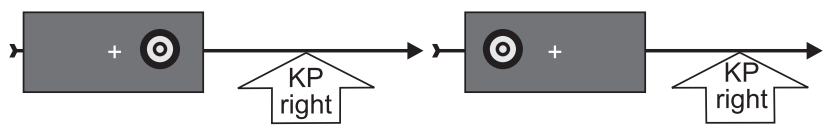

Choice- press contralateral to the stimulus

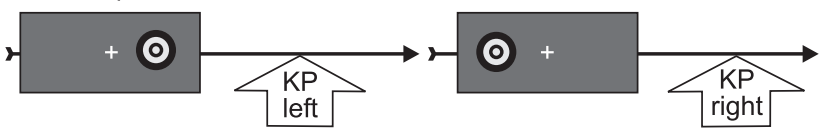

\section{Delayed Word Recognition (Word Memory) Press to repeated words

\begin{tabular}{|c|c|c|c|c|}
\hline $\begin{array}{c}\text { New } \\
\text { WORD }\end{array}$ & & $\begin{array}{l}\text { Feedback } \\
\text { TONE }\end{array}$ & $\begin{array}{c}\text { Old } \\
\text { WORD }\end{array}$ & $\begin{array}{l}\text { Feedback } \\
\text { TONE }\end{array}$ \\
\hline 300 & response & 1550 & $2600 \mathrm{~ms}$ & \\
\hline
\end{tabular}

Rhyme Judgment (Rhyme) Press to words that end in the -AY sound

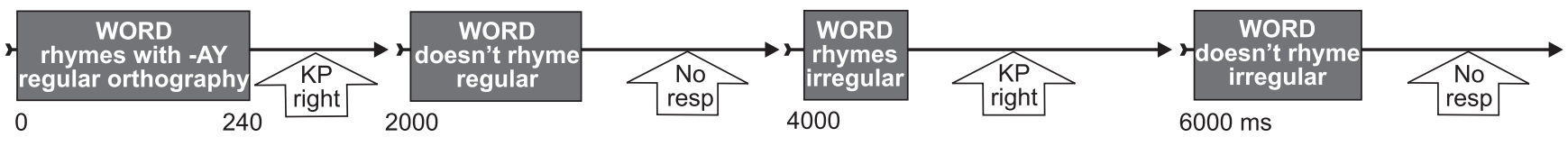

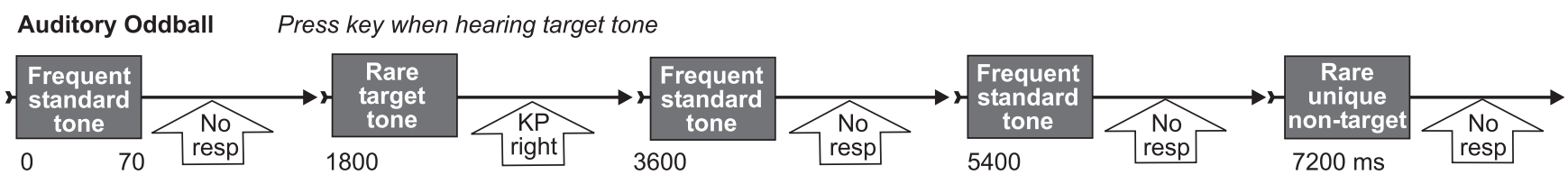

Color-Cued Conditional Letter Discrimination (Cued Conditional RT) Left KP if letter in cued color is H; Right KP if letter in cued color is $S$ All 16 possible conditions are shown below, grouped by cue and correct response. Red letter cues are indicated by italics.

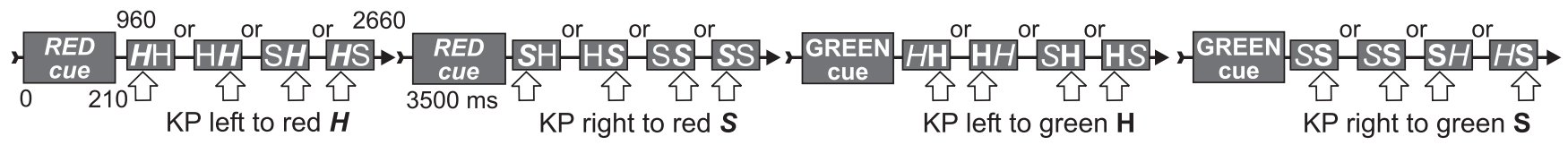

Figure 2. Five cognitive tasks and conditions compared in the present study. Time lines show the sequence of events in typical trials. KP, Key press. Please see Materials and Methods for additional explanation.

(simple/choice RT): targets flashed for $60 \mathrm{~ms}$ in the left or right visual field, and subjects responded with the left or right hand under two simple instructions (press always left or always right, regardless of stimulus laterality) and two choice instructions (press always ipsilateral or always contralateral to the stimulus). There were 196 trials for each of these four instructions. Stimulus onset asynchrony (SOA) was randomized from 1550 to 1950 ms. (2) Delayed word recognition (word memory): the subject memorizes 10 words that subsequently served as recognition targets on one-half of the trials randomized with unrepeated words. Words were visually presented for $300 \mathrm{~ms}$ in white font on a black background. There were 240 items in the test section; one-half of them were unrepeated, and one-half were 10 target words repeated 12 times randomly. Subjects were required to press a key with their dominant hand within $1200 \mathrm{~ms}$ after presentation of a repeating word. At $1550 \mathrm{~ms}$ poststimulus, a $55 \mathrm{~ms}$ sawtooth feedback tone was presented indicating whether the response (or lack thereof) had been correct $(1000 \mathrm{~Hz})$ or wrong $(200 \mathrm{~Hz})$ (Halgren et al., 1994). (3) Rhyme judgment (rhyme): the subject was requested to press a key to each word rhyming "AY" in a set of 240 words. Words differed in whether they rhymed with the target and whether they had regular orthography (e.g., "say") or irregular (e.g., "weigh"). Words were presented for $240 \mathrm{~ms}$, and SOA was $2000 \mathrm{~ms}$. (4)
Auditory oddball: subjects pressed a key to rarely occurring target tones $(76 ; 10.5 \%)$ embedded in a series of frequently occurring standard tones $(571 ; 79 \%)$ and nontarget novel tones $(76 ; 10.5 \%)$ requiring no response. All stimuli were $70 \mathrm{~ms}$ in duration, presented every $1.8 \mathrm{~s}$. Each nontarget novel sound was a unique sound differing in pitch and harmonics but with the same amplitude envelope as the pure tones serving as frequents and targets (Marinkovic et al., 2001). (5) Color-cued conditional letter discrimination (cued conditional RT): subjects were presented with a color cue for $210 \mathrm{~ms}$ ("red" or "green"). After a delay of $750 \mathrm{~ms}$, two letters ( $\mathrm{HH}, \mathrm{SS}, \mathrm{SH}$, or HS) in two colors were presented for $1700 \mathrm{~ms}$. If the letter in the cued color was an $\mathrm{H}$, then the subject made a left-handed keypress; if the letter in the cued color was an S, then the subject made a right-handed keypress (Gehring and Knight, 2000). The 16 permitted combinations of cues and imperative stimuli with the correct responses are shown in Figure 3, bottom panel. There were a total of 533 trials. Total SOA was $3500 \mathrm{~ms}$. Only Pt2 was tested on task 5.

Adequate performance was found in most tasks for both subjects (Table 1). When available, error rates were lower than $7 \%$ except for Pt1 on the rhyme judgment task (37.5\% errors). Mean reaction times ranged from 449 to $912 \mathrm{~ms}$. 
Table 1. Behavioral results

\begin{tabular}{rlllll}
\hline & $\begin{array}{l}\text { Simple/choice } \\
\text { RT }\end{array}$ & $\begin{array}{l}\text { Word } \\
\text { memory }\end{array}$ & $\begin{array}{l}\text { Auditory } \\
\text { oddball }\end{array}$ & Rhyme & $\begin{array}{l}\text { Cued } \\
\text { conditional RT }\end{array}$ \\
\hline Pt1 & & & & & \\
RT & N/A & N/A & $659(151)$ & $912(288)$ & $\mathrm{N} / \mathrm{G}$ \\
ER & N/A & $3.75 \%$ & $6.58 \%$ & $37.50 \%$ & $\mathrm{~N} / \mathrm{G}$ \\
Pt2 & & & & & \\
RT & $449(76)$ & $784(96)$ & $\mathrm{N} / \mathrm{A}$ & $819(129)$ & $\mathrm{N} / \mathrm{A}$ \\
ER & $1.65 \%$ & $4.17 \%$ & $\mathrm{~N} / \mathrm{A}$ & $0.42 \%$ & $\mathrm{~N} / \mathrm{A}$ \\
\hline
\end{tabular}

For RT, SDs are in parentheses. ER, Error rate; $N / A$, not available as a result of technical problems; $N / G$, task not given to that patient.

\section{Results}

Extensive task-related activity was found in all sites and tasks. Activity was measured as CSD, which is the transmembrane current density. EPSCs produce current sinks at the active synapses, with passive sources as current returns (Nicholson and Freeman, 1975). At the membrane potentials typical of waking neocortex (Destexhe et al., 2003), IPSCs should produce current sources at the active synapses, with passive sinks as current returns. If simultaneous MUA increases during a current sink, then it probably represents an EPSC. Conversely, if MUA decreases during a current source, then it probably represents active inhibition, an IPSC.

In all four recording sites, the strongest CSD responses occurred from $\sim 300$ to $800 \mathrm{~ms}$ after stimulus onset and were located in superficial layers. In Pt1 left ACC and Pt2 both left and right ACC, this response was a current source, followed from $\sim 800$ to $1400 \mathrm{~ms}$ by a current sink. In both locations with MUA recordings, superficial cell firing decreased during the local source, suggesting active inhibition. Pt1 right ACC and Pt2 left ACC also generated task-related theta activity in superficial layers. Theta in Pt 2 showed a task-related transient increase in phase locking to distant cortical sites.

CSD recordings from two patients during multiple tasks are shown in Figure 3. Recordings in Figure $3 a$ show a large source (upward deflection) that is evoked from $\sim 300$ to $800 \mathrm{~ms}$ after stimulus onset by visual targets (in a simple/choice reaction time task), words (in declarative memory and rhyming tasks), and brief sounds (in an auditory oddball task). The currents are significantly larger to stimuli that provoked wrong responses in the simple/choice RT task, as well as to feedback tones indicating a wrong response in the word memory task. The currents are larger to rare stimuli in the auditory oddball task and to old (i.e., repeated) words in the word memory task. The currents are larger when the response requires a choice in the simple/choice RT task, or the word orthography is irregular in the rhyming task. All responses exhibit similar morphologies and time courses and were recorded at the same microcontact. They show that the same ACC microdomain can respond in very similar ways to quite different tasks and stimuli, with differential responses to errors (either indicated by feedback or not), to rare events (presumably evoking orienting responses), to repeated words, and to difficulty (in stimulus-response mapping or orthographic-phonological decoding).

Biophysically, the source in the top panel could be attributable to active inhibitory synapses or could represent a passive current return to excitatory synapses located elsewhere. In Figure $3 b$, simultaneous MUA and CSD recordings from patient 2 allows the net local level of excitation to be estimated. A source is again observed across multiple tasks. The technical quality of the recordings is not as good, and relatively little task related modula- tion occurs between conditions. However, MUA significantly decreases during the CSD source in the auditory oddball, word memory, and rhyming tasks, suggesting that the source may represent active inhibition.

In Figure $3 a$, the cortical layer in which the source is located is hard to determine, because the laminar probe was in the sulcus (i.e., was not perpendicular to cortical surface). In Figure $3 b$, the source was recorded in the most medial contacts of the laminar probe, suggesting upper layers, but the technical quality of the recording was not adequate to confirm this suggestion.

Both the superficial location of the source and the association of the source with decreased MUA are confirmed in recordings from the left ACC of Pt2, as shown in Figure 4. The MRI indicated that the probe penetrated the crown of the gyrus perpendicular to its surface. At the top, CSD sources (blue) and sinks (red) over the cortical depth are plotted as contours versus time. At the bottom are plotted CSD and local MUA waveforms from a medial laminar contact near the cortical surface. A repeated observation across most tasks and conditions is a superficial sink from $\sim 200$ to $800 \mathrm{~ms}$ poststimulus, displayed as a blue area in the contour plots and an upward deflection in the CSD waveforms. The source is accompanied by a sink in deeper layers (red area in contour plots) with approximately the same time course. Simultaneous MUA is inhibited in all tasks and conditions, again with a similar time course. In most cases, the superficial source inverts to a strong sink after $\sim 800 \mathrm{~ms}$. Note that the sources in both Pt1 left ACC (Fig. 3a) and Pt2 right ACC (Fig. 3b) also invert to sinks after $\sim 800 \mathrm{~ms}$. These recordings also confirm the responsiveness of ACC microdomains to multiple tasks and the modulation of this response by different condition contrasts, including difficulty in stimulus-response mapping, word repetition, and cue consistency. Thus, across these three ACC microdomains, and across multiple tasks and conditions, a superficial CSD source with decreased MUA indicate active inhibitory postsynaptic currents.

The fourth recording location (Pt1 right ACC) (Fig. 5) also shows a strong CSD response across multiple tasks with large differentiations across task conditions. Again, the response is larger to choice than simple RT, to wrong than correct trials in that task, to repeated than nonrepeated words, and to rare auditory stimuli. The CSD contour has a different pattern than that seen in the other sites described above: rather than a source in the superficial layers, there is a sink. This sink is also different in beginning $\sim 100-200 \mathrm{~ms}$ later than the source seen in the other three sites. In most cases, the initial activity was a smaller sink in middle layers, beginning shortly after $100 \mathrm{~ms}$. This pattern and timing is more typical of what has been observed in laminar recordings from temporal and prefrontal neocortical sites (E. Halgren, C. Wang, I. Ulbert, S. Knake, K. Marinkovic, J. Wu, J. Madsen, and D. Schomer, unpublished observations). This observation may indicate that only some ACC microdomains show the superficial inhibitory pattern that appears to characterize the recordings described above. Alternatively, this microcontact array may not have penetrated to the cortical surface. In that case, a slightly deeper penetration may have recorded a superficial source indicating inhibition as in the other sites.

The CSD responses described above were based on averaging the CSD with respect to stimulus onset. This method eliminates activity that is not phase locked to stimulus onset. Because the ACC generates theta activity in animals, and it has been proposed that the ERN is actually averaged theta activity in humans (Luu et al., 2003), we examined the ongoing CSD and noted strong oscillations in the theta range in Pt1 right ACC and Pt2 left ACC (Fig. 6). Aver- 
aging the CSD on the peaks of these oscillations revealed an average frequency of $\sim 4-5$ $\mathrm{Hz}$ (Fig. 6a1,b1). In both cases, theta was generated mainly in superficial layers (Fig. $6 a 2, b 2)$. Theta power was related to the stimulus, increasing after the stimulus and showing significantly different responses to old versus new words and to rare versus frequent auditory stimuli (Fig. 6a3). Theta did not have any strong or obvious effect on the MUA in the period between tasks (Fig. 6b3). When averaged during the peri-stimulus epoch, theta was associated with MUA decrease (Fig. 6b3) but without a theta-range periodicity, suggesting that this decrease was unrelated to the theta but rather was attributable to other aspects of the response (compare Fig. 4).

The consistency of the phase of theta band activity was estimated between the ACC and a sample of temporal and frontal lobe sites using potential gradient measurements (Fig. 7). Phase locking significantly increased in a task-related manner, including a consistent increase from $\sim 200$ to $700 \mathrm{~ms}$ after the stimulus. Additional peaks between $\sim 500$ and $\sim 1000 \mathrm{~ms}$ were also observed. These transient increases in the similarity of the local field potential were found between both right and left ACC and sites in temporal neocortex (near the superior temporal sulcus), the frontal neocortex (including inferior, middle, and superior frontal gyri and the orbital gyrus), as well as mesial temporal hippocampal and parahippocampal leads. The increases were apparent both when comparing ACC to ipsilateral cortical as well as contralateral cortical sites. No consistent increases in ACC spectral power or ACCneocortical phase locking were observed outside of the theta band.

In summary, the most common response was a current source and MUA decrease in ACC superficial layers from $\sim 300$ to $800 \mathrm{~ms}$ after onset, followed by a current sink. Theta rhythm was also found in superficial layers. These responses were observed across a variety of tasks, occurred during different phases of tasks, such as cue, stimulus, and feedback, and modulated across multiple task conditions. These responses were recorded in very small regions, corresponding to parts of one or two cortical columns.

\section{Discussion}

Multiple cognitive correlates in single ACC microdomains

A clear finding of this study was that synaptic currents and neuronal firing recorded at individual ACC locations responded to multiple tasks and contrasts within tasks. These responses reflected activity within $\sim 300 \mu \mathrm{m}$ from the recording site, as a result of the small
CSD Pt1 Left ACC $\quad$ CSD
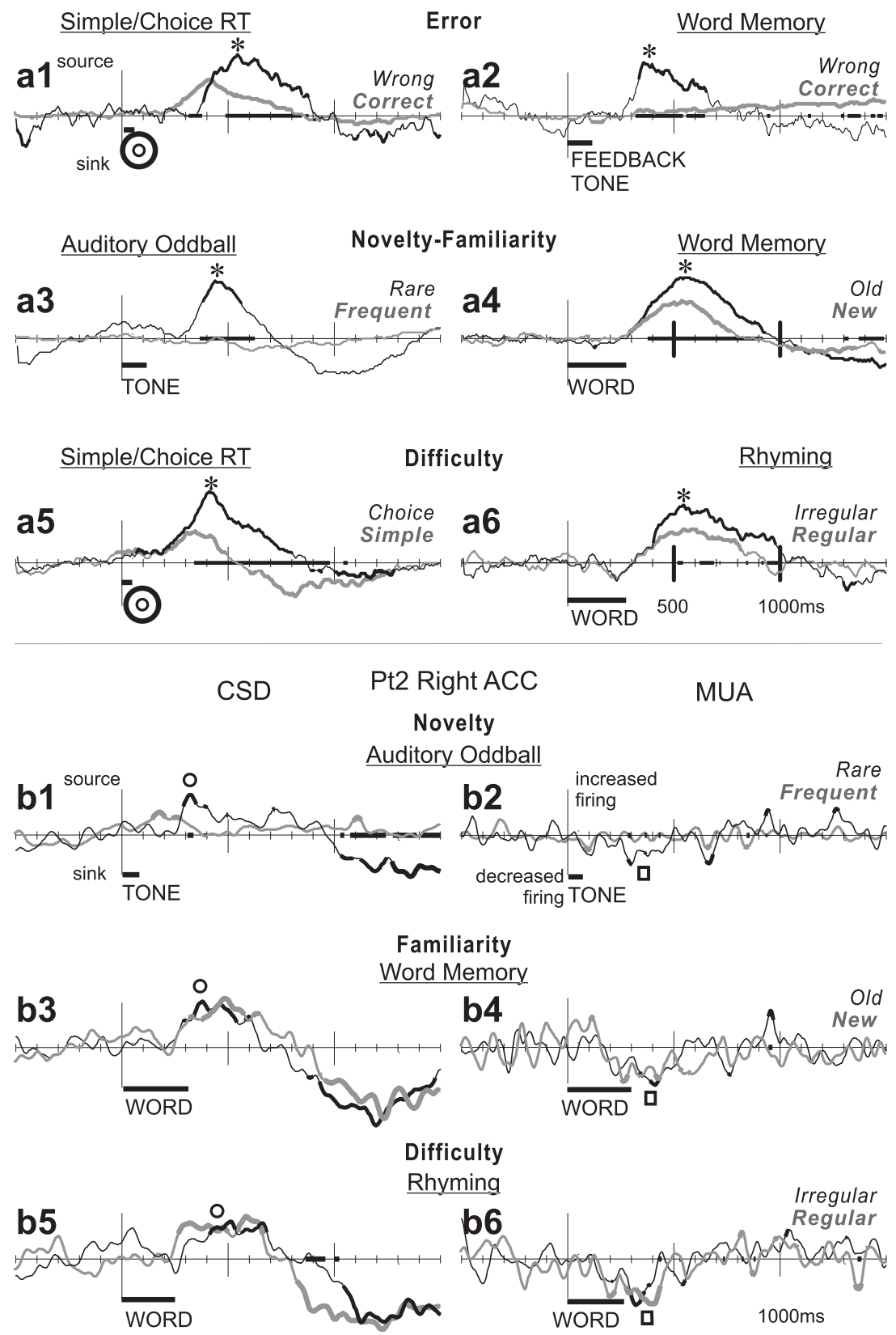

Figure 3. Possible IPSC indicated by a large CSD source with decreased MUA across multiple tasks. a, CSD waveforms recorded from the left ACC of Pt1. A large current source $\left.{ }^{*}\right)$ was evoked from $\sim 300$ to $800 \mathrm{~ms}$ in simple/choice RT (a1, a5), word recognition memory (a2, a4), auditory oddball (a3), and rhyming (a6). Source currents were larger after wrong (compared with correct) responses (a1) and after a feedback tone indicating wrong responses (a2), suggesting modulation by errors; to rare than frequent tones (a3) and to old rather than new words (a4), suggesting modulation by novelty-familiarity; to stimuli that required a differential choice response [compared with a constant simple response (a5)]; or an evaluation of irregular orthography [as opposed to regular orthography (a6)], suggesting a relationship to difficulty, perhaps in stimulus-response mapping.b, CSD (left) and simultaneous MUA (right) waveforms recorded from the right ACC of Pt2. Again, a current source $(\bigcirc)$ was evoked from $\sim 300$ to $700 \mathrm{~ms}$ in a variety of tasks involving auditory discrimination (b1), word recognition memory (b3), or rhyming (b5), although differentiation between task conditions is not as clear. Simultaneous MUA recordings show a decrease during these tasks $(\square)$ from $\sim 200$ to $500 \mathrm{~ms}(b 2, b 4,66)$. Task names are underlined; condition names are in italics. Dark gray bars below $x$-axes indicate stimulus presentation periods. The $x$-axis is thickened when the two conditions are significantly different from each other; CSD and MUA waveforms are thickened when significantly different from zero (two-tailed $p<0.01$ ).

size and close spacing of the recording contacts and the CSD/ MUA analytic methods used. Comparably high spatial resolution has also been reported for ultrahigh field (7 T) fMRI in animals (Yang et al., 1998). However, in the usual hemodynamic studies, 

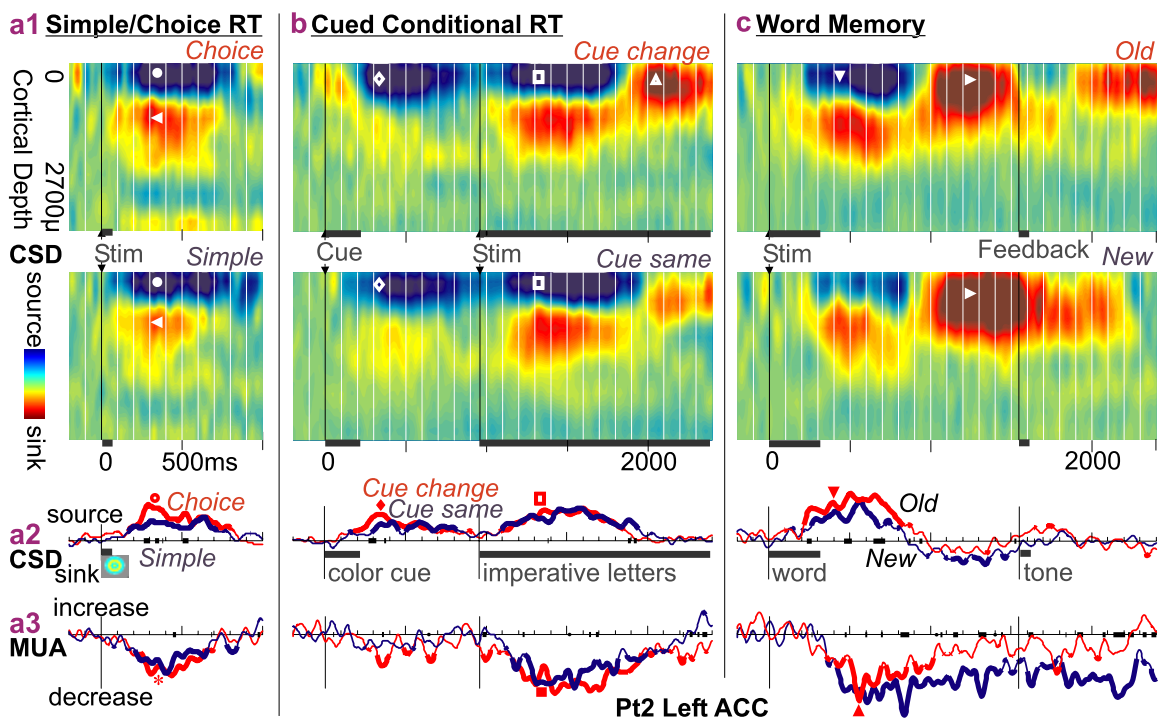

Figure 4. Possible IPSCs in superficial cortical layers across multiple cognitive tasks; Pt2 left ACC. a, CSD and MUA during simple and choice responses to lateralized visual stimuli. a1, Contour plots of CSD over time and cortical depth show sustained superficial sources (blue region with $\bullet$, where positive current leaves the cell) with deeper sinks (red region with $\mathbf{4}$, where positive current enters the cell). a2, The same data are plotted as a waveform from a superficial contact, showing a larger source $(\bigcirc)$ to choice than simple RT. a3, MUA decreases $\left({ }^{*}\right)$ during the sources shown in $a 1$ and $a 2$, indicating that they are likely IPSC $s . b$, A cued conditional letter discrimination task evokes again a strong superficial source $(\diamond)$ that is slightly larger when the cue is different from the preceding trial ( $\bullet$ ). The source evoked by the imperative stimulus $(\square)$ is accompanied by decreased MUA $(\square)$ and is followed by a superficial sink $(\triangle)$. C, A similar pattern of superficial source $(\boldsymbol{\nabla})$ followed by a sink $(\boldsymbol{\nabla})$ and MUA inhibition $(\boldsymbol{\Delta})$ is evoked by words in a memory task, with a larger source to the repeated (old) words. Note that to visualize all of the responses, the vertical scale in the left column has been compressed twofold. Thickening of the CSD and MUA waveforms indicates that they are significantly different from zero; thickening of the $x$-axis indicates that the two conditions are significantly different from each other (two-tailed $p<0.01$ ).

spatial resolution is limited by voxel size and spatial smoothing to a volume $\sim 1000 \times$ larger than that of CSD/MUA. Spatial resolution of hemodynamic measures is also limited by diffusion, vascular control unit size, and downstream vascular effects. Noninvasive electromagnetic measures have worse spatial resolution, especially for extended sources, caused by uncertainties inherent in source estimation (Dale and Halgren, 2001). Depth EEG measurements have less uncertainty but are still limited by the large contact size $\left(\sim 1 \mathrm{~mm}^{2}\right)$, intercontact distance $(\sim 3-5 \mathrm{~mm})$, and use of LFP, which, unlike CSD, can volume conduct for centimeters (Goff et al., 1978).

The current results thus establish that within the very small sampling volumes of CSD/MUA, ACC synaptic and neuronal activity can show multiple cognitive correlates. Conversely, it is still likely that there is relative specialization of different ACC areas for different functions; other ACC microdomains would presumably also respond to multiple tasks but may have a distinct profile. Indeed, although the sites had similar responses in that they all responded to multiple tasks and differentially to multiple condition contrasts, with sustained synaptic activity in superficial cortical layers, they also had clear differences in their degrees and distribution of task modulation. More studies are needed to determine the topography and individuality of these responses.

In any case, these findings clearly support the exploration of functional models in which the ACC calculates its contribution to behavior by integrating multiple types and domains of information within individual cortical columns and/or between nearby columns. That is, within the range of tasks sampled, these data favor solutions to the multiplicity of ACC activation that posit broad participation through a global function rather than multiple more restricted contributions that are topographically segregated.

The actual tasks and situations evoking ACC responses and differential responses would be consistent with a variety of different formulations for ACC function. Perhaps most consistent with the largest number of observations would be the detection of situations in which there is a larger possibility of error ("conflict monitoring"), which might be expected also to evoke an orienting response (Table 2). However, the activation of a structure in a particular task does not imply that it is essential for performance of that task, nor even that it makes a significant contribution to the task. Given the involvement of the ACC in multiple anatomical systems, it is also possible that some activation reflects a collateral involvement that is only tangentially related to the intended behavioral manipulation. Furthermore, the current study had no effective probe of ACC neuronal output, because most ACC efferents arise from deep layers where we did not record MUA (Barbas, 2000).

\section{ACC generation of error-related brain potentials}

A negative scalp potential termed "N2" occurs just before the response in conflict situations, and an "error-related negativity" (ERN) peaks $\sim 120 \mathrm{~ms}$ after initiation of an incorrect response during speeded tasks (Gehring et al., 1993; Dehaene et al., 1994). The association of similar situations with ACC hemodynamic activation, as well as their general scalp topography (Holroyd et al., 1998; Scheffers and Coles, 2000), have led some to suggest that N2 and ERN are generated in ACC (Falkenstein et al., 2000; van Veen and Carter, 2002). Less ambiguous localization has been obtained from direct intracranial ACC recordings showing LFP during incorrect responses (Halgren et al., 2002). The current study provides additional confirmation that ACC generates ERPs during the same period as the N2 and ERN, with similar task correlates. However, more study is needed, including simultaneous scalp and laminar recordings during key tasks and detailed biophysical modeling to conclude that the scalp-recorded N2 and ERN are generated, in whole or in part, in ACC.

In other studies, ACC has been identified as one of the principal cortical structures generating potentials correlated with the orienting response (Baudena et al., 1995). The P3a, elicited by novel stimuli, evokes an involuntary reorientation of attention as well as a constellation of autonomic features including a prominent electrodermal response (Marinkovic et al., 2001). Such features may be mediated by ACC projections directly to brainstem autonomic efferent structures, and ACC stimulation can provoke autonomic and general changes in cortical tonus (Devinsky et al., 1995). Neuroimaging studies also found ACC activation in orientation or autonomic control, in particular to rare stimuli in 
oddball tasks (Braver et al., 2001; Downar et al., 2001; Kiehl et al., 2001). The current study provides additional evidence for a role of the ACC in integrating cortical with autonomic aspects of phasic arousal to cognitive stimuli.

\section{ACC generation of theta}

Task-related theta activity was visible in the spontaneous CSD in the ACC of both patients. The theta rhythm is a dominant EEG feature of limbic structures including the hippocampal formation and cingulate gyrus (Leung and Borst, 1987) in most mammals, where it occurs during nonautomatic movements (Vanderwolf, 1969) and orienting (Grastyan et al., 1966). It also entrains location-specific unit activity in rats (O'Keefe and Nadel, 1978), a property that has been hypothesized to aid in memory retrieval (McNaughton, 1998). The task correlates of the mammalian theta thus resemble in several respects those associated with ACC hemodynamic activation (reviewed above) as well as those found in the current study to differentially evoke human ACC synaptic and unit activity.

Theta in the human scalp EEG has also been related to memory processes (Bastiaansen and Hagoort, 2003). The scalp topography of a prominent "frontal midline theta" during working memory tasks is consistent with generation in ACC (Gevins et al., 1997; Ishii et al., 1999), and scalp theta power is correlated across subjects with ACC glucose metabolic rate (measured with PET) (Pizzagalli et al., 2003). Subdural grid recordings have found task-related theta in numerous locations over the cortical convexity (Raghavachari et al., 2001). A single case report recorded spontaneous theta activity in the vicinity of ACC (Uchida et al., 2003). The current findings provide more direct and unambiguous support for task-related theta generation in the human ACC. Furthermore, the task-related increase in phase locking observed here indicates that the ACC theta forms part of a larger network involving widespread cortical locations in the temporal, frontal, and possibly other areas, consistent with the widespread coactivation of ACC with other areas observed with fMRI (Kiehl et al., 2000, 2001).

It is interesting to contrast the current results with depth recordings from the fusiform gyrus during the same word memory task as used in the current study and a similar task using faces. As in the current study, stimuli evoked an event-related increase in spectral power (Klopp et al., 1999). However, unlike the current study, the increase was wideband (from 5 to $45 \mathrm{~Hz}$ ) rather than restricted to the theta range,

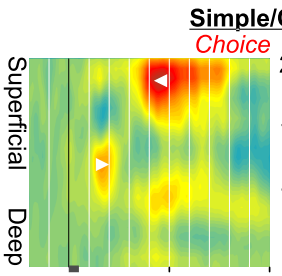

a1 Simple
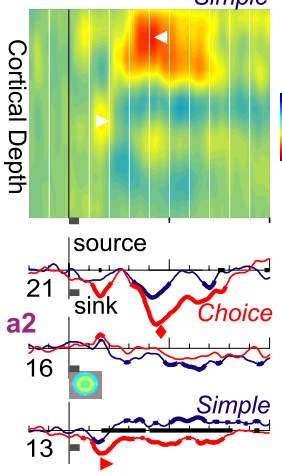
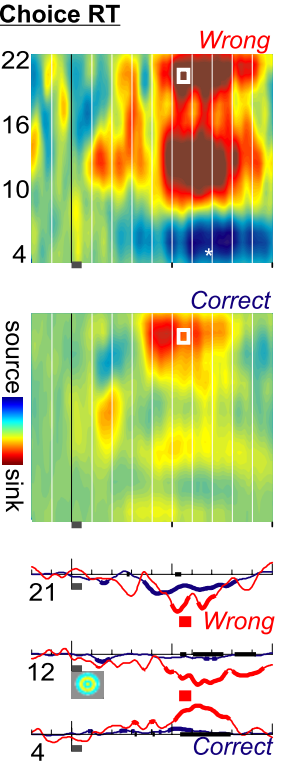

Pt1 Right ACC

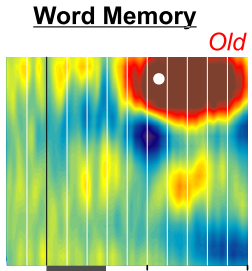

b1
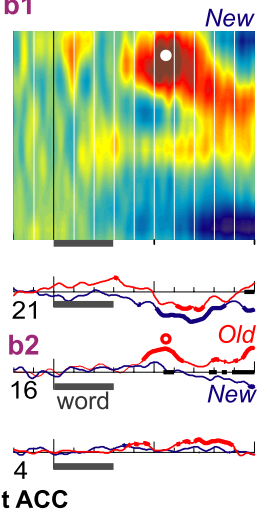

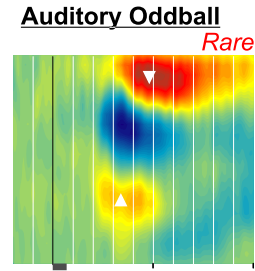

c1

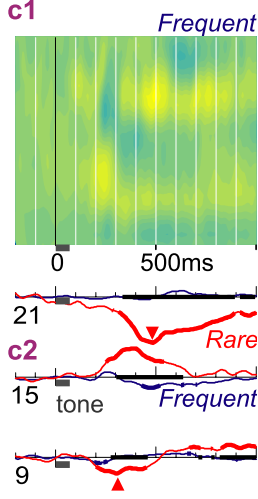

Figure 5. CSD sinks in cognitive tasks. CSD contours (above) and waveforms (below) in the right ACC of Pt1, where, unlike other sites, the CSD was dominated by sinks rather than sources. a1, CSD contours recorded as the subject makes simple and choice responses to lateralized visual stimuli. The earliest response seen in this site is a small sink in middle layers between 100 and 200 $\mathrm{ms}(\boldsymbol{)})$, followed by a larger sink in superficial layers from $\sim 350$ to $800 \mathrm{~ms}(\boldsymbol{)})$. The response to choice responses is larger than to simple responses. A larger difference is seen when the trials with incorrect responses are segregated from those with correct responses $(\square)$. The deep source $\left(^{*}\right)$ may be a passive return current. $a 2$, CSD waveforms from selected channels in the different conditions show the larger sinks to choice $(\bullet)$ and wrong $(\square)$ trials. b1, A superficial sink $(\bullet)$ is also evoked by words in a declarative memory task from $\sim 350$ to $1000 \mathrm{~ms}$, with a larger response to repeated words. b2, Traces from selected channels again show that differential responses $(\bigcirc)$ are present in multiple layers of ACC. c1, c2, Current sinks initially in middle layers $(\boldsymbol{\Delta})$ then more superficially $(\boldsymbol{\nabla})$ from $\sim 300$ to $1000 \mathrm{~ms}$ are again prominently evoked, in this case by rare tones. Note that to visualize all of the responses, the vertical scale in the left column has been compressed two times in the second and third columns and four times in the right column. CSD waveforms are thickened when significantly different from zero; the $x$-axis is thickened when the two conditions are significantly different from each other (two-tailed $p<0.01$ ).
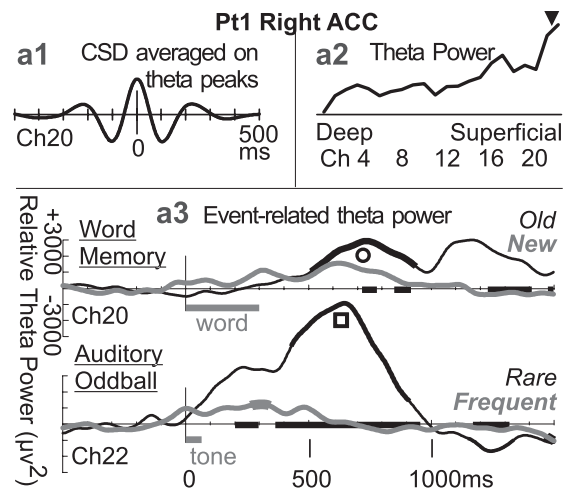

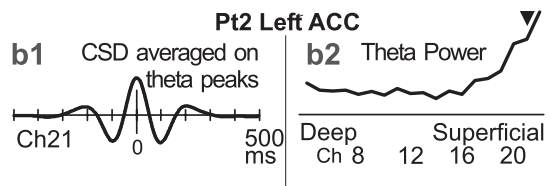

b3 MUA averaged on theta peaks

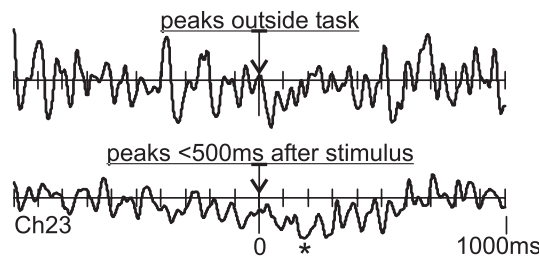

Figure 6. Task-related theta rhythm generated in superficial cortical layers of ACC of two patients. a1, b1, When averaged on the peak of the single sweep LFP, the CSD theta showed a period of $\sim 200 \mathrm{~ms}$ in both patients. a2, b2, Theta power (4-7 Hz) was calculated on single-sweep CSD from -512 ms before to $1536 \mathrm{~ms}$ after stimulus onset in simple/choice RT. In both patients, theta power was concentrated in superficial cortical layers $(\boldsymbol{\nabla})$. a3, Event-related theta power was calculated from individual trial CSD recorded in the superficial layers of the right ACC in Pt1 and then averaged. It showed a strong task-related increase peaking at $\sim 700 \mathrm{~ms}$ after words (O), especially when repeated words, and after tones $(\square)$, especially when infrequent. The $x$-axis is thickened when the two conditions are significantly different from each other; the waveforms are thickened when significantly different from zero (two-tailed $p<0.01$ ). b3, MUA recorded in the superficial layers of Pt2 left ACC was averaged with respect to peaks of the local theta rhythm. No relationship is apparent during the period between tasks, but a decreased firing is apparent with respect to theta occurring immediately after the stimulus $(*)$. was specific for faces rather than occurring to a variety of stimuli, was early and brief (150-210 ms) rather than later and extended ( 200-1000 ms), and was followed by a profound decrease in spectral power rather than being a monophasic increase. In con- 

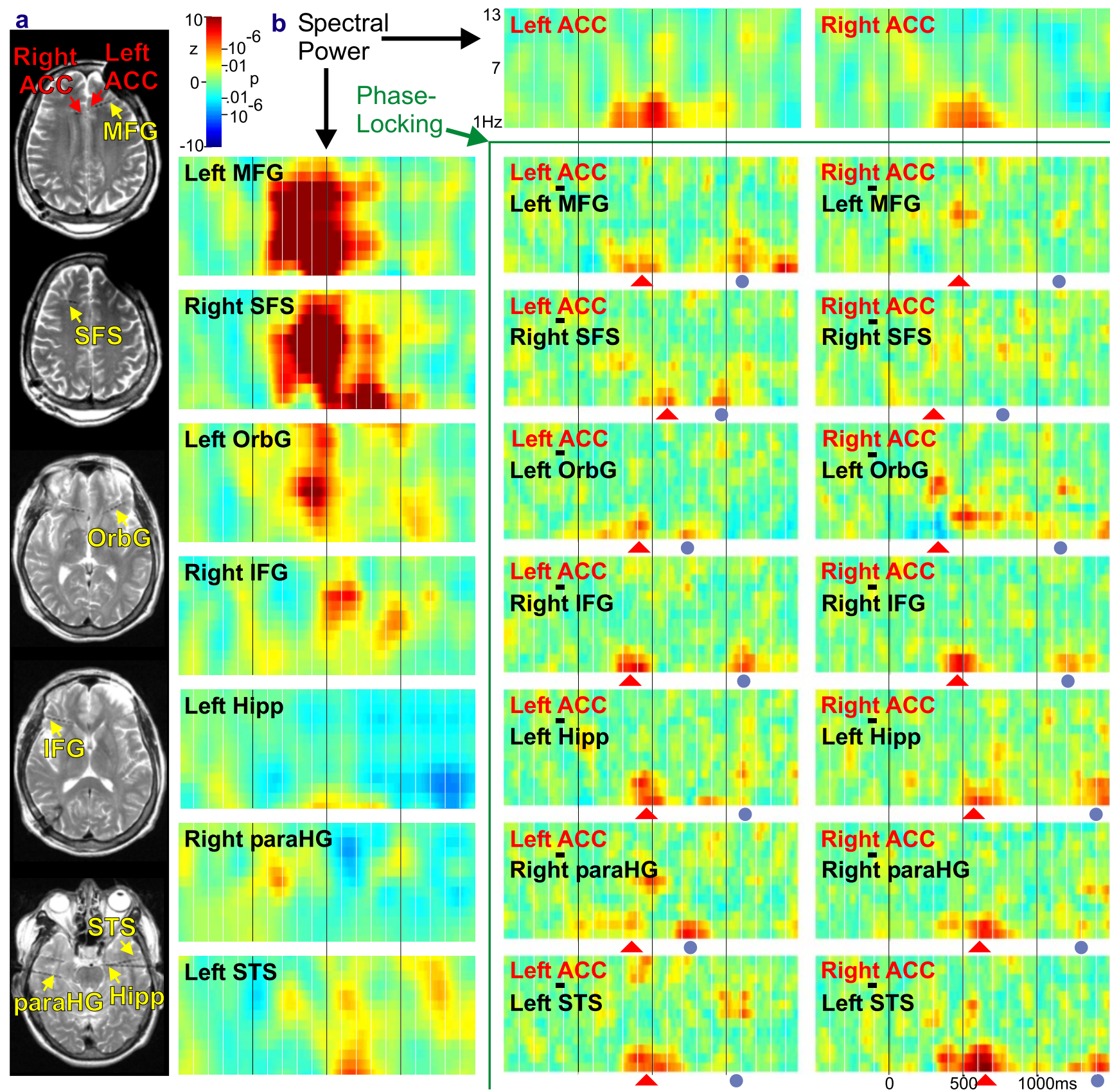

Figure 7. Single trial spectral analysis of ACC interaction with other cortical sites. $a$, The locations of micro laminar contacts in ACC (labeled in red) and macro contacts in the frontal and temporal lobes (labeled in yellow) seen in MRI obtained with the probes in place. The defect over the left frontal area is an artifact caused by an external connector. IFG, Inferior frontal gyrus; MFG, middle frontal gyrus; SFS, superior frontal sulcus; OrbG, orbital gyrus; Hipp, hippocampus; STS, superior temporal sulcus; paraHG, parahippocampal gyrus. $b$, Interactions were calculated between micro and macro contacts. Each colored box plots $z$-scores comparing spectral measures for each frequency (from 1 to $13 \mathrm{~Hz}$; $y$-axis) and each latency ( -500 - $1500 \mathrm{~ms}$; $x$-axis) for every trial to those calculated in the baseline period. Spectral power during the rhyme task is plotted in the boxes on the top row and left column; phase locking is plotted in the boxes at bottom right (other tasks gave similar results). Across sites, the most consistent event-related spectral changes were in the theta and gamma bands. Task-related increases in phase locking in these bands between both ACC and multiple frontotemporal sites occurred most reliably between 200 and $700 \mathrm{~ms}$ after stimulus onset (indicated by $\mathbf{\Delta}$ ). A second, less consistent burst of phase locking occurred at $\sim 1000 \mathrm{~ms}$ (indicated by $\mathbf{\text { ) }}$. The correspondence of the z-scores to probabilities in the normal distribution are shown on the scale.

trast to the fusiform gyrus but similar to the current results, prefrontal sites showed only a late low-frequency $(5-12 \mathrm{~Hz})$ spectral power increase to both faces and words. Again, similar to the current study, faces evoked a phasic increase in coherence between the fusiform gyrus and multiple neocortical sites in the temporal, parietal, and frontal lobes (Klopp et al., 2000). However, unlike the current study, the coherence increase was brief and confined to the higher-frequency bands. Based on this limited sample, one may speculate that the increased gamma- frequency power and coherence that has been hypothesized to promote binding of sensory elements into a percept is characteristic of sensory cortices, whereas high-level association may synchronize through theta range activity (von Stein and Sarnthein, 2000).

Inhibition in superficial layers

The most consistent task-related neuronal response observed here is a current source with unit firing decrease indicating IPSCs 
Table 2. Consistency of putative ACC functions with observed CSD/MUA responses

\begin{tabular}{|c|c|c|c|c|c|c|c|c|c|}
\hline \multirow[b]{2}{*}{ Evoking tasks } & \multirow[b]{2}{*}{$\begin{array}{l}\text { Comparison } \\
\text { conditions }\end{array}$} & \multirow[b]{2}{*}{$\begin{array}{l}\text { Phase } \\
\text { of task }\end{array}$} & \multirow{2}{*}{$\begin{array}{l}\text { Effectiveness } \\
\text { in evoking } \\
\text { responses }\end{array}$} & \multicolumn{6}{|c|}{ Putative function } \\
\hline & & & & $\begin{array}{l}\text { Error } \\
\text { detection }\end{array}$ & $\begin{array}{l}\text { Conflict } \\
\text { monitoring }\end{array}$ & $\begin{array}{l}\text { Orienting } \\
\text { response }\end{array}$ & Memory & $\begin{array}{l}\text { Motor } \\
\text { control }\end{array}$ & $\begin{array}{l}\text { Stimulus-response } \\
\text { mapping }\end{array}$ \\
\hline Simple/ choice RT & Correct/ wrong & Stimulus & ** & $* *$ & $*$ & * & & ** & \\
\hline Word memory & Correct/ wrong & Feedback & $* *$ & $* *$ & * & $* *$ & & & \\
\hline Simple/ choice RT & Choice/ simple & Stimulus & $* *$ & & $* *$ & $*$ & & ** & $* *$ \\
\hline Auditory oddball & Rare/ frequent & Stimulus & ** & & $*$ & $* *$ & & & $*$ \\
\hline Word memory & New/ old & Stimulus & * & & * & $*$ & ** & $*$ & \\
\hline Cued conditional RT & Cue change/ cue same & Cue & * & & $*$ & $*$ & & & $* *$ \\
\hline Rhyme & Irregular/ regular & Stimulus & * & & $*$ & * & & & * \\
\hline
\end{tabular}

The number of asterisks indicates the relative strength.

in superficial ACC layers. One possible source for this inhibition could be local GABAergic interneurons, whose neuropil (as indicated by parvalbumin and calbindin labeling) is most dense in layer III of ACC (Nimchinsky et al., 1997). A second possible source is cholinergic fibers from the $\mathrm{Ch} 4$ cell group in the nucleus basalis of Meynert that project mainly to superficial ACC in primates and humans (Geula and Mesulam, 1989; Lewis, 1991; Selden et al., 1998). This is the same system as the medial septum/ diagonal band of Broca, whose cholinergic and GABAergic projections to multiple limbic and neocortical sites produce the theta rhythm (Bland and Oddie, 1998). The proximal cause of the hippocampal theta in rats appears to be $\mathrm{Cl}^{-}$-mediated IPSCs (Leung, 1998). Although the local neuronal mechanisms of the ACC theta are less clear, AMPA infusion into the nucleus basalis results in decreased cingulate glucose metabolism, suggesting that these basal forebrain influences may be mainly inhibitory (Browne et al., 1998). Consistent with this interpretation, in the current recordings, the theta rhythm originated mostly from ACC superficial layers, and during task performance appeared to be associated with decreased firing. However, the overall effect of cholinergic or theta modulation is a change in the mode of information processing, which extends beyond simple inhibition.

It is unlikely that ACC inhibition is attributable directly to input from dorsolateral prefrontal or other isocortex, which project to the deep layers of ACC (Barbas, 2000). However, such input could occur indirectly via projections to ACC interneurons and/or to basal forebrain modulatory structures. One would predict such projections, because ACC superficial inhibition distinguishes between error and correct trials, and the ERN does not make such distinctions in patients with prefrontal lesions (Gehring and Knight, 2000).

In conclusion, the current results are most consistent with modulation of superficial ACC layers across a wide variety of tasks and situations. This modulation may arise in the basal forebrain, under the control of prefrontal and other areas, and be mediated by local interneurons. It starts well before the behavioral response and continues for several hundred milliseconds. It is larger when the task is difficult or an error has been made. We hypothesize that this modulatory influence gates ACC output during behavior. Although firing from the ACC output layers was not recorded, evidence for ACC-neocortical interaction during this period was found in a transient increase in the phase locking of their synaptic activity in the theta range. Inhibition in cortical pyramidal cells is commonly followed by activation of a nonspecific cation channel that is suppressed by normal waking levels of depolarization (Destexhe et al., 2003). In the present recordings, the superficial source is followed by a superficial sink, suggesting that rebound from superficial inhibition could prime the ACC to deal with the consequences of action in the postresponse period.

\section{References}

Allman JM, Hakeem A, Erwin JM, Nimchinsky E, Hof P (2001) The anterior cingulate cortex. The evolution of an interface between emotion and cognition. Ann NY Acad Sci 935:107-117.

Barbas H (2000) Connections underlying the synthesis of cognition, memory, and emotion in primate prefrontal cortices. Brain Res Bull 52:319-330.

Bastiaansen M, Hagoort P (2003) Event-induced theta responses as a window on the dynamics of memory. Cortex 39:967-992.

Baudena P, Heit G, Clarke JM, Halgren E (1995) Intracerebral potentials to rare target and distractor auditory and visual stimuli: 3. Frontal cortex. Electroencephalogr Clin Neurophysiol 94:251-264.

Bland BH, Oddie SD (1998) Anatomical, electrophysiological and pharmacological studies of ascending brainstem hippocampal synchronizing pathways. Neurosci Biobehav Rev 22:259-273.

Braver TS, Barch DM, Gray JR, Molfese DL, Snyder A (2001) Anterior cingulate cortex and response conflict: effects of frequency, inhibition and errors. Cereb Cortex 11:825-836.

Browne SE, Muir JL, Robbins TW, Page KJ, Everitt BJ, McCulloch J (1998) The cerebral metabolic effects of manipulating glutamatergic systems within the basal forebrain in conscious rats. Eur J Neurosci 10:649-663.

Bunge SA, Ochsner KN, Desmond JE, Glover GH, Gabrieli JD (2001) Prefrontal regions involved in keeping information in and out of mind. Brain 124:2074-2086.

Bush G, Luu P, Posner MI (2000) Cognitive and emotional influences in anterior cingulate cortex. Trends Cogn Sci 4:215-222.

Cabeza R, Dolcos F, Prince SE, Rice HJ, Weissman DH, Nyberg L (2003) Attention-related activity during episodic memory retrieval: a crossfunction fMRI study. Neuropsychologia 41:390-399.

Carter CS, Macdonald AM, Botvinick M, Ross LL, Stenger VA, Noll D, Cohen JD (2000) Parsing executive processes: strategic vs. evaluative functions of the anterior cingulate cortex. Proc Natl Acad Sci USA 97:1944-1948.

Corbetta M, Akbudak E, Conturo TE, Snyder AZ, Ollinger JM, Drury HA, Linenweber MR, Petersen SE, Raichle ME, Van Essen DC, Shulman GL (1998) A common network of functional areas for attention and eye movements. Neuron 21:761-773.

Crosson B, Sadek JR, Bobholz JA, Gokcay D, Mohr CM, Leonard CM, Maron L, Auerbach EJ, Browd SR, Freeman AJ, Briggs RW (1999) Activity in the paracingulate and cingulate sulci during word generation: an fMRI study of functional anatomy. Cereb Cortex 9:307-316.

Dale AM, Halgren E (2001) Spatiotemporal mapping of brain activity by integration of multiple imaging modalities. Curr Opin Neurobiol 11:202-208.

Dehaene S, Posner MI, Tucker DM (1994) Localization of a neural system for error detection and compensation. Psychol Sci 5:303-305.

Destexhe A, Rudolph M, Pare D (2003) The high-conductance state of neocortical neurons in vivo. Nat Rev Neurosci 4:739-751.

Devinsky O, Morrell MJ, Vogt BA (1995) Contributions of anterior cingulate cortex to behavior. Brain 118:279-306.

Downar J, Crawley AP, Mikulis DJ, Davis KD (2001) The effect of task relevance on the cortical response to changes in visual and auditory stimuli: an event-related fMRI study. NeuroImage 14:1256-1267.

Dum RP, Strick PL (1993) The cingulate motor areas. In: Neurobiology of cingulate cortex and limbic thalamus (Vogt BA, Gabriel M, eds), pp 415441. Boston: Birkhäuser. 
Dum RP, Strick PL (2002) Motor areas in the frontal lobe of the primate. Physiol Behav 77:677-682.

Duncan J, Owen AM (2000) Common regions of the human frontal lobe recruited by diverse cognitive demands. Trends Neurosci 23:475-483.

Falkenstein M, Hoormann J, Christ S, Hohnsbein J (2000) ERP components on reaction errors and their functional significance: a tutorial. Biol Psychol 51:87-107.

Fiez JA, Petersen SE (1998) Neuroimaging studies of word reading. Proc Natl Acad Sci USA 95:914-921.

Frith CD, Friston K, Liddle PF, Frackowiak RS (1991) Willed action and the prefrontal cortex in man: a study with PET. Proc R Soc Lond B Biol Sci 244:241-246.

Gehring WJ, Knight RT (2000) Prefrontal-cingulate interactions in action monitoring. Nat Neurosci 3:516-520.

Gehring WJ, Goss B, Coles MGH, Meyer DE, Donchin E (1993) A neural system for error detection and compensation. Psychol Sci 4:385-390.

Geula C, Mesulam MM (1989) Cortical cholinergic fibers in aging and Alzheimer's disease: a morphometric study. Neuroscience 33:469-481.

Gevins A, Smith ME, McEvoy L, Yu D (1997) High-resolution EEG mapping of cortical activation related to working memory: effects of task difficulty, type of processing, and practice. Cereb Cortex 7:374-385.

Goff WR, Allison T, Vaughan Jr HG (1978) The functional neuroanatomy of event related potentials. In: Event-related brain potentials in man (Callaway E, Tueting P, Koslow SH, eds), pp 1-79. New York: Academic.

Grastyan E, Karmos G, Vereczkey L, Kellenyi L (1966) The hippocampal electrical correlates of the homeostatic regulation of motivation. Electroencephalogr Clin Neurophysiol 21:34-53.

Grover FS, Buchwald JS (1970) Correlation of cell size with amplitude of background fast activity in specific brain nuclei. J Neurophysiol 33:160-171.

Halgren E, Baudena P, Heit G, Clarke JM, Marinkovic K (1994) Spatiotemporal stages in face and word processing. 1. Depth-recorded potentials in the human occipital, temporal and parietal lobes. J Physiol (Paris) 88:1-50.

Halgren E, Boujon C, Clarke J, Wang C, Chauvel P (2002) Rapid distributed fronto-parieto-occipital processing stages during working memory in humans. Cereb Cortex 12:710-728.

Holroyd C, Dien J, Coles M (1998) Error-related scalp potentials elicited by hand and foot movements: evidence for an output-independent errorprocessing system in humans. Neurosci Lett 242:65-68.

Humphrey DR (1968) Re-analysis of the antidromic cortical response. II. On the contribution of cell discharge and PSPs to the evoked potentials. Electroencephalogr Clin Neurophysiol 25:421-442.

Ishii R, Shinosaki K, Ukai S, Inouye T, Ishihara T, Yoshimine T, Hirabuki N, Asada H, Kihara T, Robinson SE, Takeda M (1999) Medial prefrontal cortex generates frontal midline theta rhythm. NeuroReport 10:675-679.

Kiehl KA, Liddle PF, Hopfinger JB (2000) Error processing and the rostral anterior cingulate: an event-related fMRI study. Psychophysiology 37:216-223.

Kiehl KA, Laurens KR, Duty TL, Forster BB, Liddle PF (2001) Neural sources involved in auditory target detection and novelty processing: an event-related fMRI study. Psychophysiology 38:133-142.

Klopp J, Marinkovic K, Chauvel P, Nenov V, Halgren E (2000) Early widespread cortical distribution of coherent fusiform face activity. Hum Brain Mapp 11:286-293.

Klopp JC, Halgren E, Marinkovic K, Nenov VI (1999) Face-selective eventrelated spectral changes in the human fusiform gyrus. Clin Neurophysiol 110:677-683.

Kollias SS, Alkadhi H, Jaermann T, Crelier G, Hepp-Reymond MC (2001) Identification of multiple nonprimary motor cortical areas with simple movements. Brain Res Brain Res Rev 36:185-195.

Kronland-Martinet R, Morlet J, Grossmann A (1987) Analysis of sound patterns through wavelet transforms. Intern J Pattern Recognit Artif Intell 1:273-302.

Lachaux JP, Rodriguez E, Martinerie J, Varela FJ (1999) Measuring phase synchrony in brain signals. Hum Brain Mapp 8:194-208.

Leung LS (1998) Generation of theta and gamma rhythms in the hippocampus. Neurosci Biobehav Rev 22:275-290.

Leung LW, Borst JG (1987) Electrical activity of the cingulate cortex. I. Generating mechanisms and relations to behavior. Brain Res 407:68-80.

Lewis DA (1991) Distribution of choline acetyltransferase-immunoreactive axons in monkey frontal cortex. Neuroscience 40:363-374.
Luu P, Tucker DM, Derryberry D, Reed M, Poulsen C (2003) Electrophysiological responses to errors and feedback in the process of action regulation. Psychol Sci 14:47-53.

Marinkovic K, Halgren E, Maltzman I (2001) Arousal-related P3a to novel auditory stimuli is abolished by a moderately low alcohol dose. Alcohol Alcohol 36:529-539.

McNaughton BL (1998) The neurophysiology of reminiscence. Neurobiol Learn Mem 70:252-267.

Mesulam MM (1981) A cortical network for directed attention and unilateral neglect. Ann Neurol 10:309-325.

Nicholson C, Freeman JA (1975) Theory of current source density analysis and determination of the conductivity tensor for anuran cerebellum. J Neurophysiol 38:356-368.

Nimchinsky EA, Vogt BA, Morrison JH, Hof PR (1997) Neurofilament and calcium-binding proteins in the human cingulate cortex. J Comp Neurol 384:597-620.

Nyberg L (1998) Mapping episodic memory. Behav Brain Res 90:107-114.

O'Keefe J, Nadel L (1978) The hippocampus as a cognitive map. Oxford: Clarendon.

Paus T (2001) Primate anterior cingulate cortex: where motor control, drive and cognition interface. Nat Rev Neurosci 2:417-424.

Petersen SE, Fox PT, Posner MI, Raichle ME, Mintun MA (1989) Positron emission tomographic studies of the processing of single words. J Cogn Neurosci 1:153-170.

Peterson BS, Skudlarski P, Gatenby JC, Zhang H, Anderson AW, Gore JC (1999) An fMRI study of Stroop word-color interference: evidence for cingulate subregions subserving multiple distributed attentional systems. Biol Psychiatry 45:1237-1258.

Phan KL, Wager T, Taylor SF, Liberzon I (2002) Functional neuroanatomy of emotion: a meta-analysis of emotion activation studies in PET and fMRI. NeuroImage 16:331-348.

Picard N, Strick PL (2001) Imaging the premotor areas. Curr Opin Neurobiol 11:663-672.

Pizzagalli DA, Oakes TR, Davidson RJ (2003) Coupling of theta activity and glucose metabolism in the human rostral anterior cingulate cortex: an EEG/PET study of normal and depressed subjects. Psychophysiology 40:939-949.

Posner MI, Petersen SE, Fox PT, Raichle ME (1988) Localization of cognitive operations in the human brain. Science 240:1627-1631.

Raghavachari S, Kahana MJ, Rizzuto DS, Caplan JB, Kirschen MP, Bourgeois B, Madsen JR, Lisman JE (2001) Gating of human theta oscillations by a working memory task. J Neurosci 21:3175-3183.

Scheffers MK, Coles MG (2000) Performance monitoring in a confusing world: error-related brain activity, judgments of response accuracy, and types of errors. J Exp Psychol Hum Percept Perform 26:141-151.

Selden NR, Gitelman DR, Salamon-Murayama N, Parrish TB, Mesulam MM (1998) Trajectories of cholinergic pathways within the cerebral hemispheres of the human brain. Brain 121:2249-2257.

Uchida S, Maehara T, Hirai N, Kawai K, Shimizu H (2003) Theta oscillation in the anterior cingulate and beta-1 oscillation in the medial temporal cortices: a human case report. J Clin Neurosci 10:371-374.

Ulbert I, Halgren E, Heit G, Karmos G (2001a) Multiple microelectroderecording system for human intracortical applications. J Neurosci Methods 106:69-79.

Ulbert I, Karmos G, Heit G, Halgren E (2001b) Early discrimination of coherent versus incoherent motion by multiunit and synaptic activity in human putative MT+. Hum Brain Mapp 13:226-238.

Vanderwolf CH (1969) Hippocampal electrical activity and voluntary movement in the rat. Electroencephalogr Clin Neurophysiol 26:407-410

van Veen V, Carter CS (2002) The anterior cingulate as a conflict monitor: fMRI and ERP studies. Physiol Behav 77:477-482.

Vogt BA, Hof PR, Vogt LJ (2004) Cingulate gyrus. In: The human nervous system, Ed 2 (Paxinos G, Mai JK, eds), pp 915-949. San Diego: Elsevier Academic.

von Stein A, Sarnthein J (2000) Different frequencies for different scales of cortical integration: from local gamma to long range alpha/theta synchronization. Int J Psychophysiol 38:301-313.

Yang X, Renken R, Hyder F, Siddeek M, Greer CA, Shepherd GM, Shulman RG (1998) Dynamic mapping at the laminar level of odor-elicited responses in rat olfactory bulb by functional MRI. Proc Natl Acad Sci USA 95:7715-7720. 